\title{
Shear and compaction band formation on an elliptic yield cap
}

\author{
John W. Rudnicki \\ Department of Civil and Environmental Engineering, Northwestern University, Evanston, Illinois, USA
}

Received 16 June 2003; revised 5 December 2003; accepted 6 January 2004; published 5 March 2004.

[1] Conditions for shear and compaction localization are examined for stress states on an elliptic yield cap in the space of Mises equivalent shear stress $\bar{\tau}$ and mean normal compressive stress $\sigma$. Localization is predicted to occur when the slope of the hydrostatic stress versus inelastic volume strain curve falls to a critical value $k_{\text {crit }}$. When applied to the standard triaxial test, the analysis reveals a transition from compaction localization to shear localization as lateral confining stress $\sigma_{c}$ decreases below a particular value. This value also corresponds to the largest value of $k_{\text {crit }}$, which is zero if normality is satisfied and slightly positive if not, for either shear or compaction bands. For $\sigma_{c}$ larger than the transition value, compaction bands are the only mode of localized deformation predicted, and $k_{\text {crit }}$ becomes increasingly negative with increasing $\sigma_{c}$. For $\sigma_{c}$ smaller than the transition value, both shear bands and compaction bands are possible, but the value of $k_{\text {crit }}$ is larger for shear bands. These results are consistent with experimental observations of compaction bands on relatively flat portions of the stress versus strain curve, corresponding to $k_{\text {crit }} \approx 0$, and with their occurrence in a limited range of $\sigma_{c}$. As $\sigma_{c}$ is decreased from the transition value, the predicted angle between the normal to the shear band and the maximum compression direction increases rapidly from $0^{\circ}$ (for a compaction band) to $20^{\circ}-30^{\circ}$. This rapid increase provides a possible explanation for the infrequent observation of very low angle shear bands. INDEX TERMS: 3299 Mathematical Geophysics: General or miscellaneous; 5104 Physical Properties of Rocks: Fracture and flow; 5114 Physical Properties of Rocks: Permeability and porosity; KEYWORDS: compaction, shear bands, porosity, localization, cap surface

Citation: Rudnicki, J. W. (2004), Shear and compaction band formation on an elliptic yield cap, J. Geophys. Res., 109, B03402, doi:10.1029/2003JB002633.

\section{Introduction}

[2] Field studies in porous sandstone formations [Aydin and Johnson, 1983; Antonellini et al., 1994] have identified various forms of localized shear deformation accompanied by compaction, i.e., inelastic porosity reduction. In addition, Mollema and Antonellini [1996] have reported observations of compaction bands, planar bands of localized deformation without shear. Compaction bands have been observed to form perpendicular to the maximum compressive stress in laboratory tests on porous sandstones [Olsson, 1999; Olsson and Holcomb, 2000; Wong et al., 2001; Klein et al., 2001] and they have also been observed in a variety of other porous materials, such as metal foams [Bastawros et al., 2000; Park and Nutt, 2001], polycarbonate honeycomb [Papka and Kyriakides, 1998], snow (J. Desrues, personal communication, 2002) and ice [Kirby et al., 1992]. Similar structures appear to affect the formation of borehole breakouts in porous sandstones [Haimson, 2001; Klaetsch and Haimson, 2002]. In sandstones, field [Antonellini and Aydin, 1994, 1995; Chapin et al., 2004] and laboratory studies [Holcomb and Olsson,

Copyright 2004 by the American Geophysical Union. 0148-0227/04/2003JB002633\$09.00
2003] have shown that the permeability across both compaction bands and shear bands with significant compaction is reduced by one to two orders of magnitude. Consequently, the formation of these features in reservoirs can significantly alter the permeability structure and result in permeability barriers that adversely affect the injection or withdrawal of fluids.

[3] Olsson [1999] noted that conditions for the initiation of compaction bands could be addressed within the same framework introduced for the inception of shear bands by Rudnicki and Rice [1975]. His analysis and subsequent ones [Issen and Rudnicki, 2000, 2001; Issen, 2002; Rudnicki, 2002, 2003] demonstrated that the formation of compaction bands was possible for some stress states on a "cap" yield surface in a stress space of shear versus hydrostatic compression (Figure 1). The cap allows for inelastic behavior under purely hydrostatic conditions and a reduction of the shear stress at yield with increasing mean compressive stress. In contrast, the Mohr-Coulomb surface, for example, is open ended on the hydrostatic axis (or the closure of the surface occurs at compressive stresses too high to be relevant). Cap models have long been used in soil plasticity (see Fossum et al. [1995] for a list of references) but their introduction is often attributed to Dimaggio and Sandler [1971]. Carroll [1991] has 


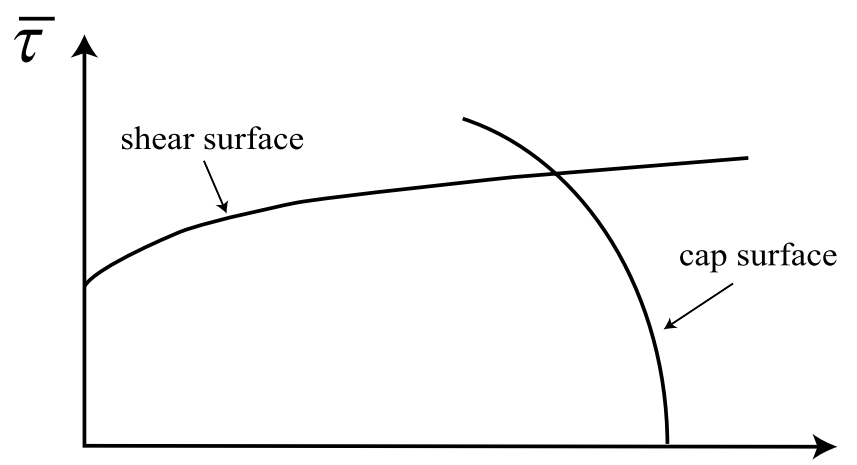

$\sigma$

Figure 1. Schematic of shear and "cap" yield surfaces in the plane of equivalent shear stress $\overline{\bar{\tau}}$ versus mean compressive stress $\sigma$.

described a related model, based on the critical state approach to modeling soil behavior [Schofield and Wroth, 1968], and has used it to describe the response of Boise sandstone. Recently, cap models have been used to describe the behavior of porous rocks [Wong et al., 1992; Olsson, 1999; Fossum and Fredrich, 2000a] and, also, porous metal powders [Gu et al., 2001]. A number of studies [Dimaggio and Sandler, 1971; Fossum and Fredrich, 1998, 2000b; Fossum et al., 1995] have found that an elliptic surface is sufficient to describe data on granular materials and porous rocks. Wong et al. [1997, 2001] have shown that elliptic surfaces describe the onset of shear enhanced compaction in a variety of sandstones. (This is the point at which the mean stress versus volumetric strain curve for an axisymmetric compression test deviates from the same curve for hydrostatic stress loading.)

[4] This paper analyzes conditions for both compaction band and shear band formation for stress states on an elliptic yield cap and applies them to the standard axisymmetric compression test. The results can be obtained by manipulation of those of Rudnicki and Rice [1975] (with the correction noted by Perrin and Leblond [1993] and discussed by Issen and Rudnicki [2000]) or by using the slightly more general results of Ottosen and Runesson [1991]. The structure of the constitutive relation used here is identical to that used by Rudnicki and Rice [1975], but it is presented in a form that is more suitable for application to a cap surface. In addition, the constitutive relation is rederived from a more general point-of-view that makes closer contact with the formulation of Ottosen and Runesson [1991]. The results are expressed in terms of predictions for a series of axisymmetric compression tests at increasing values of the lateral confining stresses.

\section{Constitutive Relation}

[5] Experimental testing of rocks has shown that there is a set of stress states for which the response can be idealized as elastic (Figure 2). For stress states on the boundary of this region, the yield surface, the deformation is nonelastic and the size and shape of this elastic region may evolve with nonelastic deformation. For simplicity, we assume that the yield surface depends on stress only through the first and second invariants (or equivalently the first invariant of the stress and the second invariant of the deviatoric stress). Detailed observations on rocks (although few in number) do show dependence on the third invariant [e.g., Kim and Lade, 1984; Lee et al., 1999]. Although this dependence is not included here, there is no fundamental impediment to doing so. The first invariant is denoted by $\sigma=-(1 / 3) \sigma_{k k}$, where the repeated subscript denotes summation and $\sigma_{k k}$ is taken to be positive in tension. The second invariant of the deviatoric stress is given by $\bar{\tau}=\sqrt{(1 / 2) s_{i j} s_{i j}}$, where the $s_{i j}=\sigma_{i j}-(1 / 3) \delta_{i j} \sigma_{k k}$ are components of the deviatoric stress and $\delta_{i j}=1$ if $i=j, \delta_{i j}=0$ if $i \neq j$. For the axisymmetric compression test with (compressive) axial stress $\sigma_{a}$ and lateral (compressive) stress $\sigma_{c}, \sigma=\left(\sigma_{a}+2 \sigma_{c}\right) / 3$ and $\bar{\tau}=\left(\sigma_{a}-\sigma_{c}\right) / \sqrt{3}$. For this test $\bar{\tau}$ and $\sigma$ can also be expressed as $\bar{\tau}=2 q / \sqrt{3}$ and $\sigma=p-q / 3$, where $q=\left(\sigma_{a}-\right.$ $\left.\sigma_{c}\right) / 2$ and $p=\left(\sigma_{a}+\sigma_{c}\right) / 2$.

[6] If inelastic deformation is assumed to depend on stress only through $\bar{\tau}$ and $\sigma$, then the yield condition can be written as

$$
F\left(\bar{\tau}, \sigma, \alpha_{\kappa}\right)=0
$$

where the $\alpha_{\kappa}$ are a set of internal variables that keep track of the history of inelastic deformation, such as microcrack density, dislocation density, inelastic porosity, etc. Often the $\alpha_{\kappa}$ are chosen to be the inelastic strains themselves or scalar combinations of them. For fixed $\alpha_{\kappa}$ the yield condition describes a surface in the $\bar{\tau}$ versus $\sigma$ plane (Figure 2). When the stress state is on the yield surface, we assume that the inelastic strain increments can be expressed in terms of a plastic potential $G\left(\tau, \sigma, \alpha_{\kappa}\right)$ :

$$
d \varepsilon_{i j}^{p}=d \lambda \frac{\partial G}{\partial \sigma_{i j}}=d \lambda\left\{\frac{s_{i j}}{2 \bar{\tau}} G_{\bar{\tau}}-\frac{1}{3} G_{\sigma} \delta_{i j}\right\}
$$

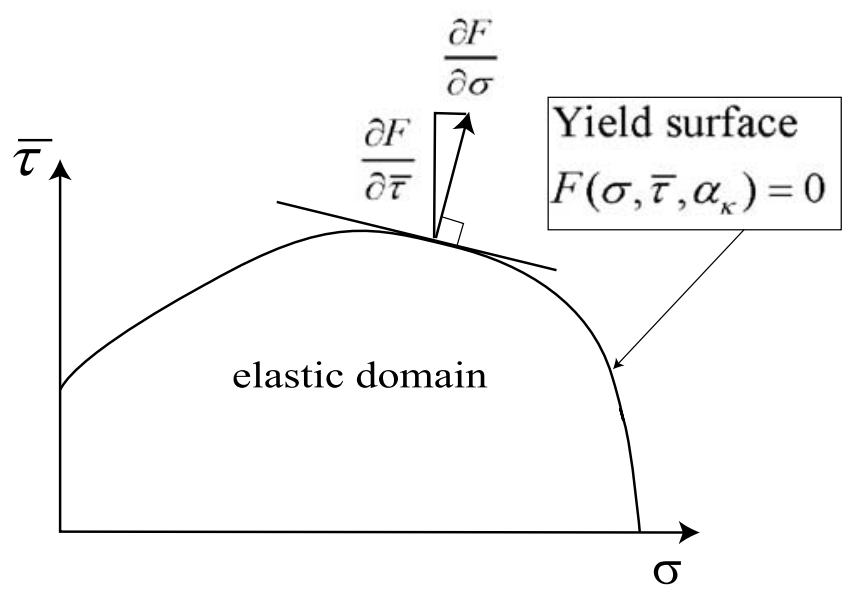

Figure 2. Schematic of the yield surface in the plane of equivalent shear stress $\bar{\tau}$ versus mean compressive stress $\sigma$. The yield surface is the boundary of the stress states for which the response is elastic and will evolve with changes in the $\alpha_{k}$, a set of parameters characterizing the current state of accumulated inelastic deformation. The normal to the yield surface $\left(F_{\sigma}, F_{\bar{\tau}}\right)$ is also shown. 


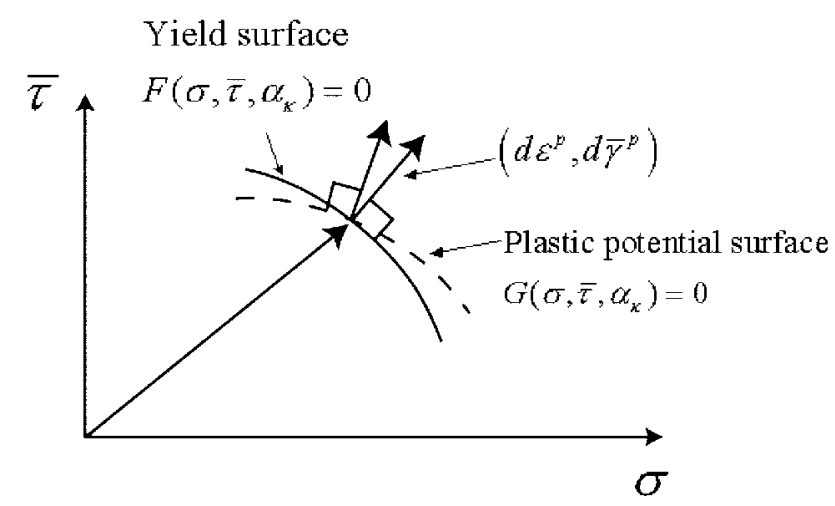

Figure 3. Schematic of the relation between the yield surface and the plastic potential surface. If these two surfaces coincide, the direction of the inelastic strain increments is normal to the yield surface.

where $G_{\bar{\tau}}=\partial G / \partial \bar{\tau}, G_{\sigma}=\partial G / \partial \sigma$ and $d \lambda$ is a nonnegative scalar. Separating the inelastic strain increment into volumetric and deviatoric contributions yields

$$
d \varepsilon_{i j}^{p}=d e_{i j}^{p}+\delta_{i j} d \varepsilon_{k k}^{p} / 3
$$

and it follows from equation (2) that

$$
d \bar{\gamma}^{p}=d \lambda G_{\bar{\tau}}, d \varepsilon^{p}=d \lambda G_{\sigma},
$$

where $d \bar{\gamma}^{p}=\left(2 d e_{i j}^{p} d e_{i j}^{p}\right)^{1 / 2}$ is the equivalent plastic shear strain increment and $d \varepsilon^{p}=-d \varepsilon_{k k}^{p}$ is the volume strain increment (positive in compression). Thus the plastic strain increment $\left(d \varepsilon^{p}, d \bar{\gamma}^{p}\right)$ can be represented as a vector perpendicular to the plastic potential surface in the plane $\bar{\tau}$ versus $\sigma$ (Figure 3 ).

[7] Because the stress state cannot lie outside the yield surface, the $\alpha_{\kappa}$ must change with inelastic deformation so that the stress state remains on the yield surface during inelastic loading. This requirement results in the following consistency condition:

$$
d F=\left(F_{\bar{\tau}} \frac{s_{i j}}{2 \bar{\tau}}-F_{\sigma} \frac{1}{3} \delta_{i j}\right) d \sigma_{i j}+F_{\alpha_{\kappa}} d \alpha_{\kappa}=0,
$$

where the subscripts on $F$ denote partial derivatives. The assumption that the inelastic response is rate-independent and the observation that the $\alpha_{\kappa}$ can change only when one of the $d \varepsilon_{i j}^{p}$ is nonzero constrains expressions for the increments of the $\alpha_{\kappa}$ to have the form

$$
d \alpha_{\kappa}=\sqrt{2 d \varepsilon_{i j}^{p} d \varepsilon_{i j}^{p}} \Phi_{\kappa}\left(\bar{\tau}, \sigma, \alpha_{\xi}, P_{k l}\right),
$$

where the factor 2 appears for convenience in succeeding expressions and $P_{k l}=d \varepsilon_{k} p / \sqrt{2 d \varepsilon_{m n}^{p} d \varepsilon_{m n}^{p}}$ is the "direction" of the inelastic strain increment. Thus equation (6) implies that the $\Phi_{\kappa}$ depend only on the ratio of components of $d \varepsilon_{i j}^{p}$ and not on the magnitude of the inelastic strain increment. Using equations (3) and (4) in equation (6) yields

$$
d \alpha_{\kappa}=d \lambda\left[\left(G_{\bar{\tau}}\right)^{2}+\frac{2}{3}\left(G_{\sigma}\right)^{2}\right]^{1 / 2} \Phi_{\kappa}\left(\bar{\tau}, \sigma, \alpha_{\xi}, P_{k l}\right) .
$$

Substituting equation (7) into equation (5), solving for $d \lambda$, and substituting the result into equation (2) yields the following expression for the plastic strain increments:

$$
d \varepsilon_{i j}^{p}=\frac{1}{H}\left\{G_{\bar{\tau}} \frac{s_{i j}}{2 \bar{\tau}}-\frac{1}{3} G_{\sigma} \delta_{i j}\right\}\left(F_{\bar{\tau}} \frac{s_{k l}}{2 \bar{\tau}}-F_{\sigma} \frac{1}{3} \delta_{k l}\right) d \sigma_{k l},
$$

where

$$
H=-F_{\alpha_{\kappa}} \Phi_{\kappa}\left(\bar{\tau}, \sigma, \alpha_{\xi}, P_{k l}\right)\left[\left(G_{\bar{\tau}}\right)^{2}+\frac{2}{3}\left(G_{\sigma}\right)^{2}\right]^{1 / 2}
$$

is a plastic hardening modulus. For application to a "cap" it will be convenient to express $H$ in terms of the hydrostatic stress versus inelastic volume strain curve. Specializing equation (8) to pure hydrostatic compressive stress, $d \sigma_{11}=$ $d \sigma_{22}=d \sigma_{33}=-d \sigma$, yields $H=k F_{\sigma} G_{\sigma}$ where $k=d \sigma / d \varepsilon^{p}$. If the elastic bulk modulus is $K$, then the slope of the hydrostat is $k /(1+k / K)$. The slope of the axial stress versus axial strain curve (for fixed lateral confining stress) is given by $E^{p} /\left(1+E / E^{p}\right)$ where $E$ is Young's modulus and

$$
E^{p}=\frac{3 F_{\sigma} G_{\sigma} k}{\left(G_{\bar{\tau}}+G_{\sigma} / \sqrt{3}\right)\left(F_{\bar{\tau}}+F_{\sigma} / \sqrt{3}\right)} .
$$

[8] This formulation is identical to that used by Rudnicki and Rice [1975] in their study of conditions for shear localization. They, however, expressed $H$ in terms of the slope of the shear stress $\bar{\tau}$ versus inelastic shear strain $\bar{\gamma}^{p}$ curve at constant mean stress $(d \sigma=0)$. The connection to the present form is obtained by specializing equation (8) to the case of simple shear, $\tau=\sigma_{12}=\sigma_{21}$, at constant mean stress, $d \sigma=0$. Then $H=G_{\bar{\tau}} F_{\bar{\tau}} h$, where $h=d \tau / d \gamma^{p}$ and $d \gamma^{p}=2 d \varepsilon_{12}^{p}=2 d \varepsilon_{21}^{p}$.

[9] The friction coefficient (local slope of the yield surface) $\mu$ and dilatancy factor $\beta$ used by Rudnicki and Rice [1975] are given by $\mu=-F_{\sigma} / F_{\bar{\tau}}$ and $\beta=-G_{\sigma} / G_{\bar{\tau}}$. Because Rudnicki and Rice [1975] focused on shear localization in low-porosity, dilatant rocks, they restricted attention to positive values of $\mu$ and $\beta$. In this case, the yield surface is not closed on the hydrostatic $(\sigma)$ axis and no inelastic deformation is caused by purely hydrostatic stress (at least in the pressure range of interest). By considering negative values of $\mu$ and $\beta$, Issen and Rudnicki [2000, 2001] applied the formulation to the case where the yield surface is closed on the $\sigma$ axis and the inelastic volume strain is compactive, as appropriate for high-porosity, compacting rocks. For application to a cap surface the present formulation has the advantage that it avoids the singularities that occur in $\mu$ and $\beta$ near the hydrostatic axis $\bar{\tau}=0$. Unless the yield surface and plastic potential have a vertex on the hydrostatic axis, symmetry suggests that their slopes are vertical at $\bar{\tau}=$ 0 and that $F_{\bar{\tau}}=G_{\bar{\tau}}=0$ there.

[10] Ottosen and Runesson [1991] have given localization conditions for a slightly more general constitutive formulation that includes the one used by Rudnicki and Rice [1975]. In particular, they give results for anisotropic elasticity and allow for a more general dependence of the yield function and plastic potential on stress. In addition, the correction of Rudnicki and Rice [1975], noted and discussed by Perrin and Leblond [1993], can be obtained from Ottosen and 
Runesson [1991]. In terms of the notation here, the $f_{i j}$ and $g_{i j}$ of Ottosen and Runesson [1991] correspond to the quantities (...) and $\{\ldots\}$ in equation (8). Ottosen and Runesson [1991] do, however, assume that the deviatoric parts of $f_{i j}$ and $g_{i j}$ have the same principal directions and values which, here, requires that $F_{\bar{\tau}}=G_{\bar{\tau}}$. They give results for both positive and negative values of $G_{\sigma}$ and $F_{\sigma}$ which correspond to the negative of their $g_{v}$ and $f_{v}$, respectively.

\section{Conditions for Localization of Deformation}

[11] As noted earlier, conditions for the occurrence of shear bands or compaction bands can be obtained by expressing the results of Rudnicki and Rice [1975] (with the correction noted by Perrin and Leblond [1993] and discussed by Issen and Rudnicki [2000]), Ottosen and Runesson [1991], or Issen and Rudnicki [2000, 2001] in terms of $k, F_{\bar{\tau}}=G_{\bar{\tau}}, G_{\sigma}$ and $F_{\sigma}$. These are summarized here for axisymmetric compression. If

$$
F_{\sigma}+G_{\sigma}>\sqrt{3} F_{\bar{\tau}},
$$

shear bands are not possible and compaction bands are the only mode of localized deformation. If this inequality is satisfied, a compaction band is predicted if the modulus $k$ becomes less than the critical value given by

$$
\begin{aligned}
& \frac{k_{\mathrm{crit}}^{\mathrm{CB}}}{G}=\frac{(1+\nu)}{9(1-\nu)}\left\{\left(\sqrt{\frac{F_{\sigma}}{G_{\sigma}}}-\sqrt{\frac{G_{\sigma}}{F_{\sigma}}}\right)^{2}\right. \\
& \left.-\left[\left(\sqrt{\frac{F_{\sigma}}{G_{\sigma}}}+\sqrt{\frac{G_{\sigma}}{F_{\sigma}}}\right)-\sqrt{3 \frac{F_{\bar{\tau}}}{F_{\sigma}} \frac{F_{\bar{\tau}}}{G_{\sigma}}}\right]^{2}\right\},
\end{aligned}
$$

where $\nu$ is Poisson's ratio. If normality is satisfied, $F_{\sigma}=G_{\sigma}$, the first term in equation (12) is zero, $k_{\text {crit }}^{\mathrm{CB}}<0$, and consequently, the slope of the hydrostatic stress versus strain curve is predicted to be negative for formation of a compaction band. If the inequality (11) is not met, then both compaction bands and shear bands are possible. The critical value of $k$ for formation of a compaction band is still given by equation (12) and that for a shear band is given by

$$
\begin{aligned}
\frac{k_{\text {crit }}^{\mathrm{SB}}}{G}= & \frac{(1+\nu)}{9(1-\nu)}\left(\sqrt{\frac{F_{\sigma}}{G_{\sigma}}}-\sqrt{\frac{G_{\sigma}}{F_{\sigma}}}\right)^{2} \\
& -\frac{(1+\nu)}{18}\left[\left(\sqrt{\frac{F_{\sigma}}{G_{\sigma}}}+\sqrt{\frac{G_{\sigma}}{F_{\sigma}}}\right)-\sqrt{3 \frac{F_{\bar{\tau}}}{F_{\sigma}} \frac{F_{\bar{\tau}}}{G_{\sigma}}}\right]^{2} .
\end{aligned}
$$

If the inequality (11) is not met, the value given by equation (12) will be less (more negative) than that given by equation (13). Because $k$ generally decreases with inelastic deformation (at least initially), localized deformation will first be possible when it falls to the largest value of $k_{\text {crit }}$. Consequently, when both shear and compaction bands are possible, shear bands are predicted to occur first. When shear bands are possible, the band angle (the angle between the normal to the band and the direction of the most compressive stress) is given by

$$
\theta_{\text {band }}=\frac{\pi}{4}-\frac{1}{2} \arcsin \left\{1-\frac{2(1+\nu)}{3 \sqrt{3} F_{\bar{\tau}}}\left[\sqrt{3} F_{\bar{\tau}}-\left(F_{\sigma}+G_{\sigma}\right)\right]\right\} .
$$

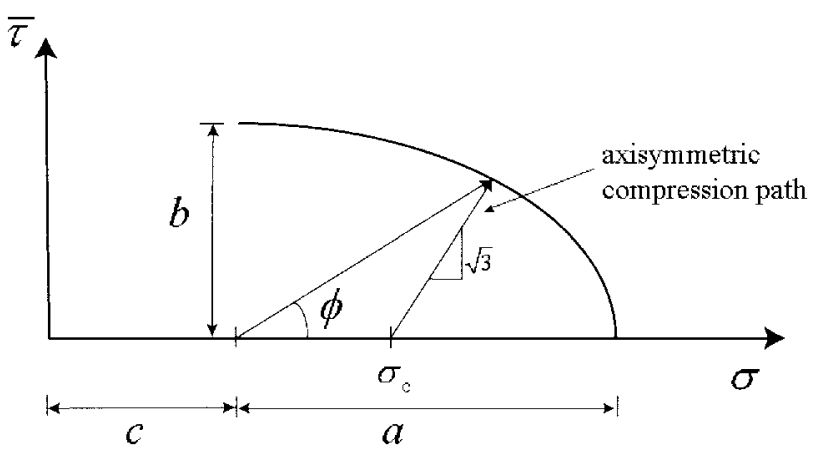

Figure 4. A quarter-elliptical cap surface with center at $\sigma=c, \bar{\tau}=0$, and semiaxes $a$ and $b$. Also shown is the path of an axisymmetric compression test following hydrostatic loading to a lateral confining stress $\sigma_{c}$.

Note that if inequality (11) is met, the argument of the arcsin is greater than one and, hence, its value is not real.

\section{Elliptic Cap Surface}

[12] An elliptic yield surface is a special case of the formulation in the preceding section. Figure 4 shows a quarter-elliptical cap surface described by

$$
F(\tau, \sigma)=\left(\frac{\sigma-c}{a}\right)^{2}+\left(\frac{\bar{\tau}}{b}\right)^{2}-1=0,
$$

where the dependence on the $\alpha_{\kappa}$ has been omitted. Thus the partial derivatives appearing in the constitutive equation (8) and the localization conditions (12), (13), and (14) are given by

$$
F_{\sigma}=2(\sigma-c) / a^{2}, \quad F_{\bar{\tau}}=2 \bar{\tau} / b^{2} .
$$

The derivatives of the plastic potential, $G_{\sigma}$ and $G_{\bar{\tau}}$, that enter equations (8), (12), (13), and (14) are assumed to be given by the first of equation (16) with $a$ replaced by $a^{\prime}$ and $G_{\bar{\tau}}=F_{\bar{\tau}}$, respectively. For stress states on the cap surface, inelastic strain increments are generally assumed to be normal to the yield surface (i.e., the plastic potential and yield surfaces coincide), but the possibility of nonnormality is included by letting $a^{\prime}$ differ from $a$. (The forms of $G_{\sigma}$ and $G_{\bar{\tau}}$ imply that the plastic potential surface is also elliptical, but stress states satisfying equation (15) will not also lie on a single elliptical plastic potential surface if normality is not satisfied. Although this complicates observational determination of the plastic potential surface, it is of no consequence for the analysis since only the gradient of the surface is needed to determine the direction of the inelastic strain increment.)

[13] Figure 4 also shows the path of a standard axisymmetric compression test. The axial and lateral stresses are simultaneously increased to $\sigma_{c}$, then the lateral stress is held fixed and the axial stress is increased. Increase of the axial stress with fixed lateral stress causes $\bar{\tau}$ and $\sigma$ to increase in the ratio $\sqrt{3}$. The aspect ratio of the ellipse is $\varepsilon=a / b$. For a given aspect ratio a point on the ellipse can be described by the angle $\phi$ or the normalized confining stress $S=\left(\sigma_{c}-c\right) /$ a. For a fixed ellipse, the angle $\phi$ decreases from $60^{\circ}$ to $0^{\circ}$ 
as the lateral confining stress increases from $\sigma_{c}=c$ to $\sigma_{c}=$ $c+a$.

[14] Wong et al. [1997] give data for the onset of "shear enhanced compaction" for several sandstones (Berea, Darley Dale, Kayenta, Rothbach 2, Adamswiller and Boise II) that are roughly described by elliptical cap surfaces. This onset is determined by the point at which the mean stress versus volumetric strain curve for an axisymmetric compression test deviates from the same curve for a hydrostatic test. Strictly speaking, these surfaces are not necessarily yield surfaces, but are likely to be similar to the initial yield surface. In addition, the shape of the surface may evolve with ongoing inelastic deformation. Thus the shape and size of the surface at the onset of either shear or compaction localization may differ from the initial surface. Nevertheless, the shape may not change drastically with deformation and, in the absence of other information, the data provide guidance in the choice of the aspect ratio $\varepsilon$. The aspect ratio is given in terms of the parameters used by Wong et al. [1997] as $\sqrt{3}(1-\gamma) / \delta$. Wong et al. [1997] state that their data are bracketed by values of $(\gamma, \delta)$ between $(0.5,0.5)$ and $(0.5,0.7)$ which correspond to values of $\varepsilon$ ranging from 1.24 to 1.73 . Klein et al. [2001] give values for Bentheim sandstone that are slightly outside this range, $\gamma=0.61$ and $\delta=0.6$, corresponding to $\varepsilon=1.126$.

[15] An analysis by Grueschow and Rudnicki [2003] of data of W. A. Olsson and D. J. Holcomb (unpublished data, 2002) from constant mean stress and hydrostatic tests on Castlegate sandstone suggests much larger values of the aspect ratio. They find an initial aspect ratio of nearly 20 since loading in shear (at constant mean stress) causes inelastic deformation almost immediately. The aspect ratio decreases to about 4 at peak stress but this value depends on the stress path.

[16] Fossum et al. [1995] use an elliptic cap to model the behavior of Salem limestone and find aspect ratios (corresponding to one third of their $R$ ) ranging from 1.67 to 2.1. These values are comparable to those determined recently by V. Vajdova et al. (Compaction, dilatancy, and failure in porous carbonate rocks, submitted to Journal of Geophysical Research, 2003) for the same rock type. Fossum and Fredrich [1998, 2000a, 2000b] derived slightly smaller aspect ratios for seven diatomite cycles from the Belridge Field (California) and three diatomites and two sands at the Lost Hills Field (California). Those for the Belridge field range from 0.47 to 1.5 with intermediate values closer to the upper end and those for the Lost Hills Field range from 0.78 to 1.70 .

\section{Results}

[17] The condition (11) defines the maximum value of $\phi$ for which compaction bands are the only mode of localized deformation that is predicted:

$$
\tan \phi<\tan \phi_{\operatorname{tran}} \equiv \frac{1+\delta^{2}}{\sqrt{3} \varepsilon^{2} \delta^{2}},
$$

where $\delta=a^{\prime} / a$ is equal to one if normality is satisfied (which differs from the $\delta$ used by Wong et al. [1997]) and, as

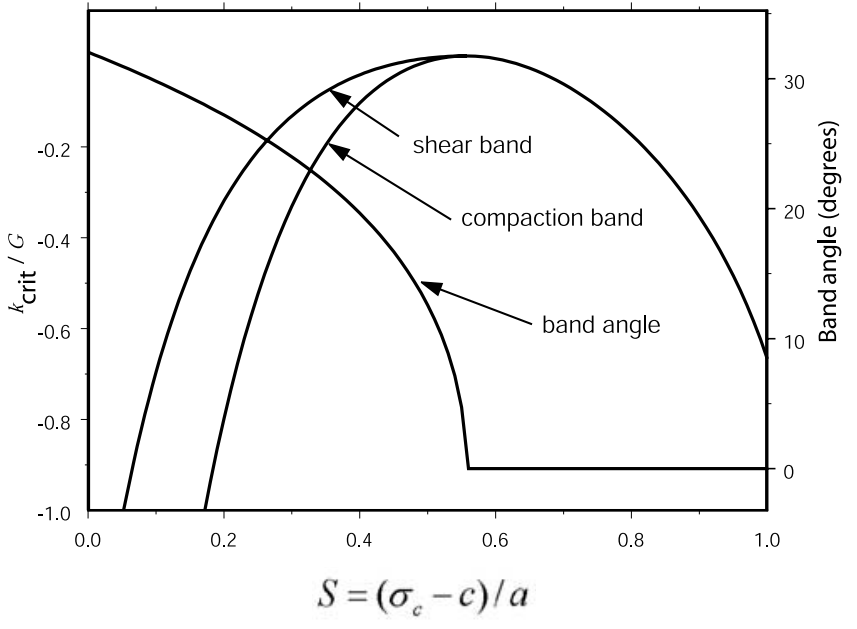

Figure 5. Critical values of $k$ for compaction bands and shear bands against the normalized confining stress $S$ for an aspect ratio $\varepsilon=1.5$ and normality $\delta=1$. The right vertical axis shows the corresponding values of the band angle.

before, $\varepsilon=a / b$ is the ellipse aspect ratio. The normalized lateral stress $S$ is given in terms of $\phi$ by

$$
S=\frac{1-(\tan \phi) / \sqrt{3}}{\sqrt{1+\varepsilon^{2} \tan ^{2} \phi}}
$$

and the inverse relation is

$$
\tan \phi=\sqrt{3} \frac{1-S \sqrt{1+3 \varepsilon^{2}\left(1-S^{2}\right)}}{1-3 \varepsilon^{2} S^{2}} .
$$

Equation (17) can be used to rewrite equation (14) as

$$
\theta_{\text {band }}=\frac{\pi}{4}-\frac{1}{2} \arcsin \left\{1-\frac{2(1+\nu)}{3}\left[1-\frac{\tan \phi_{\operatorname{tran}}}{\tan \phi}\right]\right\},
$$

where the arcsin does not have a real value when equation (17) is met (and the band angle is $0^{\circ}$ ). The right equality of equation (17) can be used to express the critical values of $k$ for compaction bands of equation (12) and shear bands of equation (13) as

$$
\frac{k_{\mathrm{crit}}^{\mathrm{CB}}}{G}=\frac{1}{9} \frac{1+\nu}{1-\nu}\left\{\left(\delta-\frac{1}{\delta}\right)^{2}-\left(\delta+\frac{1}{\delta}\right)^{2}\left[1-\frac{\tan \phi}{\tan \phi_{\text {tran }}}\right]^{2}\right\}
$$

and

$$
\frac{k_{\mathrm{crit}}^{\mathrm{SB}}}{G}=\frac{1}{9} \frac{1+\nu}{1-\nu}\left(\delta-\frac{1}{\delta}\right)^{2}-\frac{(1+\nu)}{18}\left(\delta+\frac{1}{\delta}\right)^{2}\left[1-\frac{\tan \phi}{\tan \phi_{\text {tran }}}\right]^{2} .
$$

[18] Figure 5 plots the critical values of $k$ for compaction bands and shear bands against the normalized confining stress $S$. The aspect ratio $\varepsilon=1.5$ is representative of values indicated by the data of Wong et al. [1997] and normality is assumed to be satisfied $(\delta=1)$. The righthand vertical axis gives values of the angle between the 


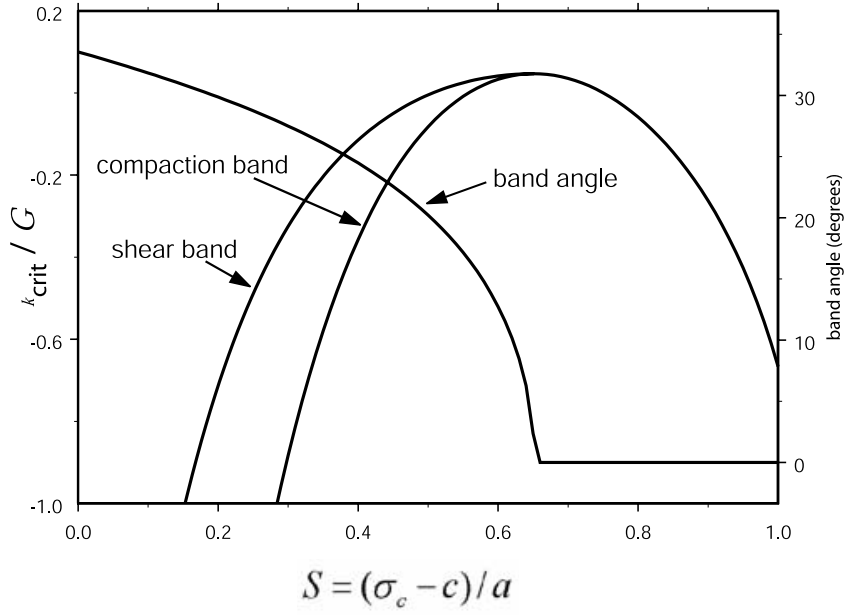

Figure 6. Same as Figure 5, except that normality is not satisfied $(\delta=1.3)$.

normal to the band and the specimen axis. The transition value of $\phi$ of equation (17) corresponds to the point where the band angle departs from $0^{\circ}$ which occurs at a value of $S$ equal to about 0.56 . For larger values of $S$ (larger values of confining stress and smaller values of $\phi$ ) compaction bands are the only mode of localized deformation that is predicted. For smaller values of $S$, both compaction bands and shear bands are possible, but, as shown in the graph, $k_{\text {crit }}^{\mathrm{SB}}>k_{\text {crit. }}^{\mathrm{CB}}$. Because normality is satisfied (the plastic potential surface is identical to the yield surface), the maximum value of $k_{\text {crit }}$ for both shear bands and compaction bands is zero and it occurs precisely at the transition between the two modes of localized deformation. Once the lateral stress is small enough so that shear bands are possible, the band angle increases rapidly: The band angle is about $20^{\circ}$ when $S$ has decreased to 0.40 .

[19] Figure 6 shows the effect of nonnormality corresponding to $\delta=1.3$. In terms of the parameters used by Rudnicki and Rice [1975], $\beta / \mu=\delta^{2}=1.69$. Nonnormality increases $k_{\text {crit }}$ for both shear and compaction bands to positive values. It also increases the value of $S$ (lateral confining stress) at which the transition from shear to compaction bands occurs to about 0.65. As in Figure 5, once the lateral stress is low enough to allow shear bands to form, the transition to band angles of $20^{\circ}-30^{\circ}$ occurs rapidly.

[20] Figure 7 shows the effect of the ellipse aspect ratio $\varepsilon$ on $k_{\text {crit }}$ and the band angle. Increasing values of $\varepsilon$ increase the lateral stress at which the transition from shear bands to compaction bands occurs. Figure 8 shows results for $k_{\text {crit }}$ and the band angle for a fixed aspect ratio $\varepsilon=1.5$ and three values of $\delta: 0.6,1.0$, and 1.4. Increasing values of $\delta$ cause the transition from shear bands to compaction bands and the maximum in $k_{\text {crit }}$ to occur at higher values of $S$. Nonnormality, corresponding to values of $\delta$ different from unity, (a)

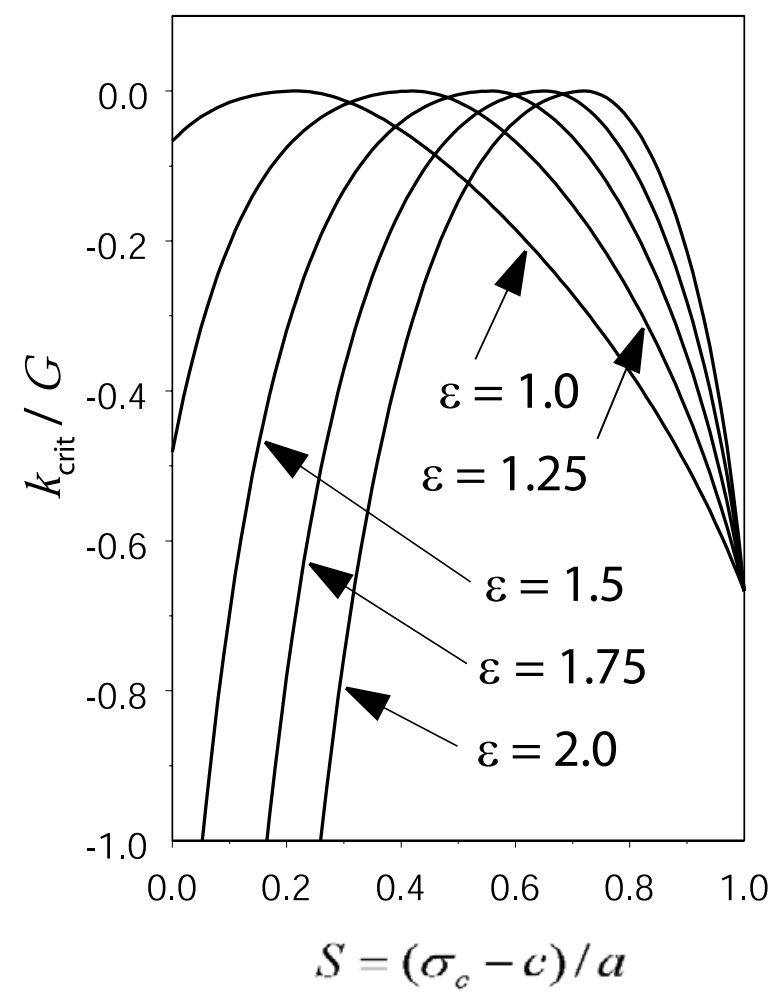

(b)

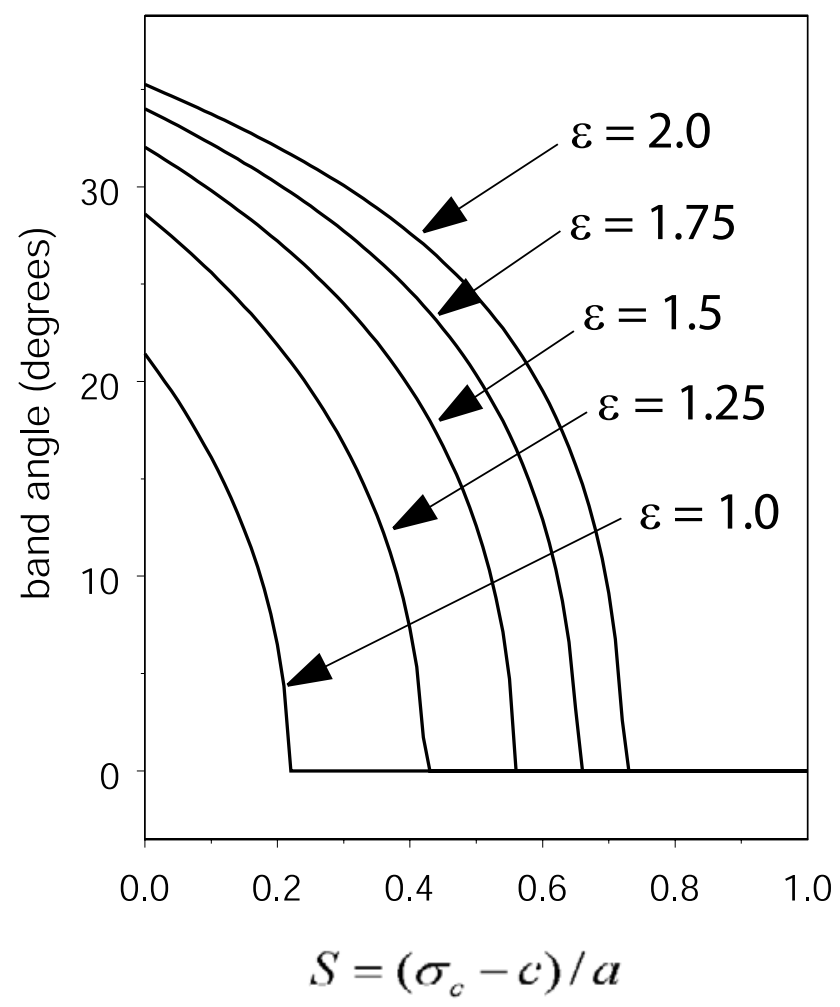

Figure 7. Effect of the aspect ratio $\varepsilon$ on (a) the critical value of $k$ and (b) the band angle for $\delta=1$. In Figure $7 \mathrm{a}$, only the larger value of $k_{\text {crit }}$ is shown when both shear and compaction bands are possible. Thus values to the left of the maximum are for shear bands, and those to the right are for compaction bands. 
(a)

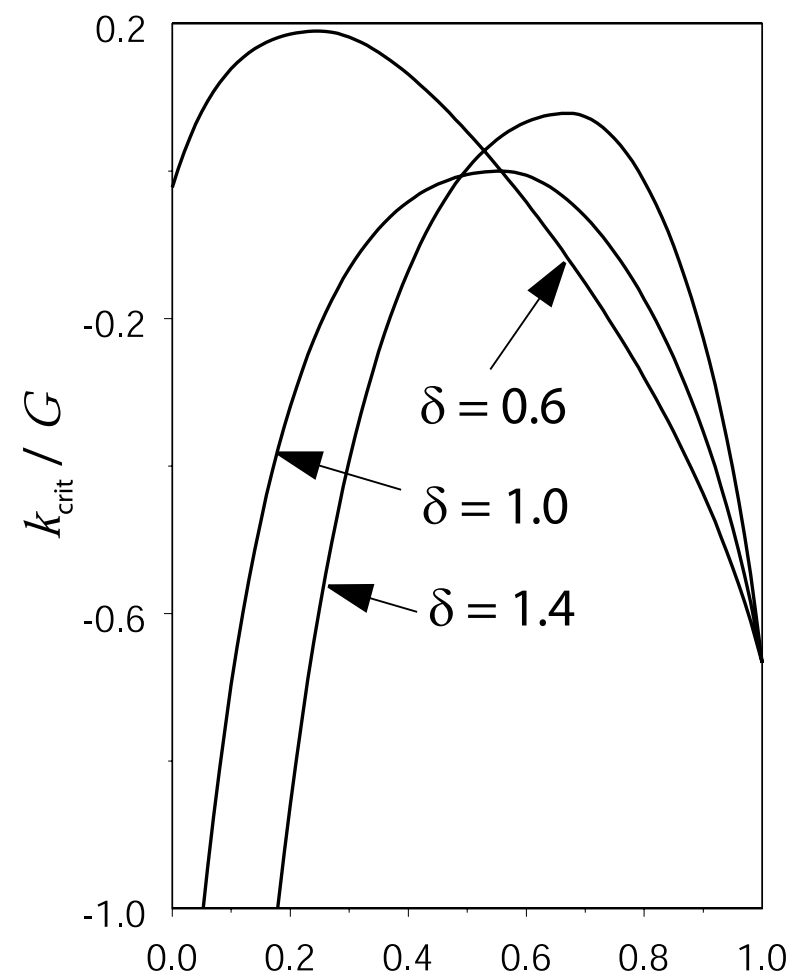

(b)

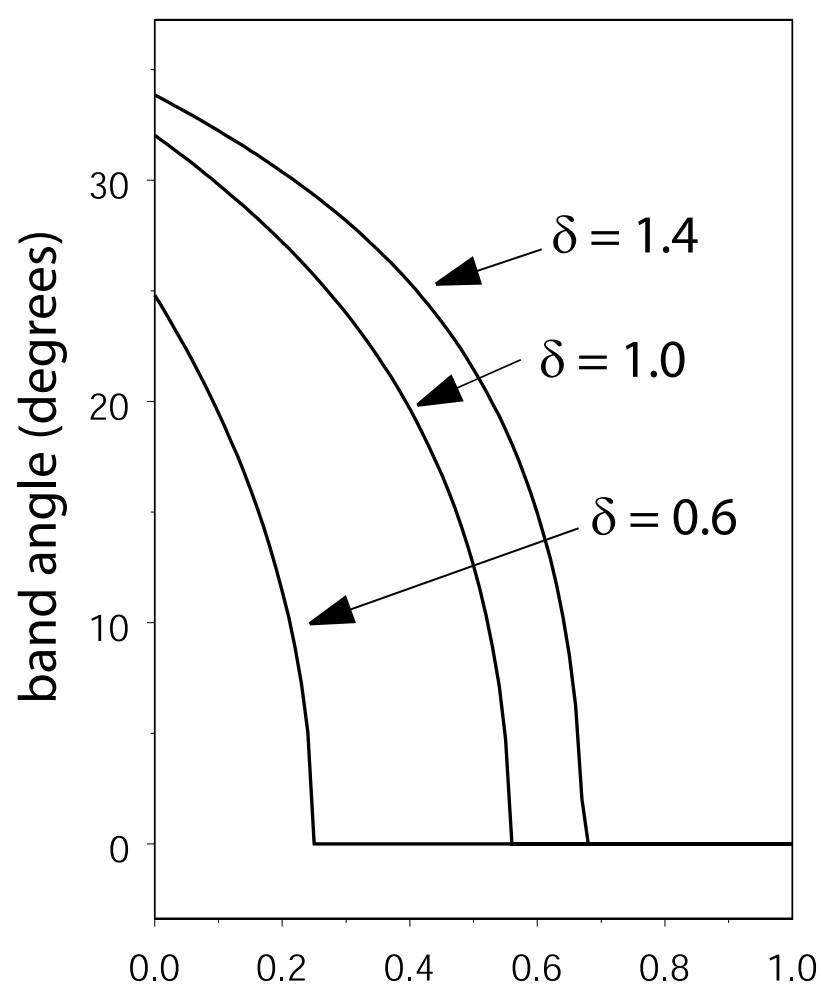

Figure 8. Effect of nonnormality on (a) $k_{\text {crit }}$ and (b) the band angle for a fixed aspect ratio $\varepsilon=1.5$.

causes the maximum value of $k_{\text {crit }}$ to be positive and values are greater for $\delta>1$ for the same value of $|\delta-1|$ Values of $\delta>(<) 1$ correspond to less (more) compaction than predicted by normality.

\section{Discussion}

[21] The predictions of this simple analysis are consistent with several features of compaction bands observed in the axisymmetric compression experiments of Baud et al. [2004] on four sandstones. They observe bands for a limited range of lateral confining stresses [Baud et al., 2004]. At confining stresses below this range shear bands are observed. At higher confining stresses (also depending on rock type), compaction bands may occur but they tend to be more diffuse. In the analysis the lower end of the range is set by the point on the ellipse at which shear bands are predicted to precede compaction bands. The analysis predicts that compaction bands are possible at higher values of the confining pressure but the value of $k_{\text {crit }}$ required becomes increasingly negative. Because this requires the slope of the mean stress versus volume strain curve to become very negative, it is unlikely that the condition for compaction band formation will be met. The analysis does assume, however, that the parameters of the ellipse are fixed. The yield surface may evolve differently depending on where the stress path intersects the initial yield surface.

[22] The value of $S$, the normalized confining stress, at which the transition from shear to compaction bands occurs is obtained by substituting $\phi_{\text {tran }}$ from the right equality of equation (17) into equation (18). This value can be con- verted to a dimensional value of the lateral confining stress if the values of the ellipse parameters $a$ and $c$ are known. Because values of $a$ and $c$ can be determined from the data of Wong et al. [1997], the calculated transition value of the confining stress can be compared with the range of confining stresses for which Baud et al. [2004] observe compaction bands for the same sandstones. The comparison is at best approximate because, as noted earlier, the surfaces defined by the data of Wong et al. [1997] correspond most closely to initial yield surfaces and the surfaces may change size and shape with the inelastic deformation that occurs between initial yield and the onset of shear or compaction localization.

[23] The parameters $a, b$, and $c$, are given in terms of those used by Wong et al. [1997] as $(1-\gamma) P^{*}, \delta P^{*} / \sqrt{3}$, and $\gamma P^{*}$, respectively, where $P^{*}$ is the grain crushing pressure under hydrostatic loading, tabulated for a variety of sandstones and some other materials in Table 4 of Wong et al. [1997]. For the Bentheim sandstone, Klein et al. [2001] give $\gamma=0.61, \delta=0.6$ and $P^{*}=390 \mathrm{MPa}$ which yields $288 \mathrm{MPa}$ for the transition value of the lateral confining stress. Baud et al. [2004] report observations of discrete compaction bands in the range of confining pressures from $120 \mathrm{MPa}$ to $395 \mathrm{MPa}$. Note that the latter, which was the highest confining pressure tested, exceeds the value of $P^{*}$ indicating at least some evolution of the yield surface. Both discrete compaction bands and high-angle shear bands were observed in a test at $90 \mathrm{MPa}$ confining pressure. Wong et al. [1997] state that their data on Berea, Rothbach and Darley Dale sandstones, also tested by Baud et al. [2004], are bracketed by values of $(\gamma, \delta)$ between $(0.5,0.5)$ and $(0.5$, 
0.7) (Values are not given for the Diemelstadt sandstone tested by Baud et al. [2004].) Values of $P^{*}$ for the Berea, Darley Dale and Rothbach sandstones are 380, 360, and $240 \mathrm{MPa}$, respectively. For the range of $(\gamma, \delta)$ given the transition confining stresses are 268-313 $\mathrm{MPa}, 254-$ $296 \mathrm{MPa}$, and 170-198 MPa for the Berea, Darley Dale and Rothbach. None of these three sandstones were tested at confining stresses as high as the predicted transition value. Only for the Berea sandstone were discrete compaction bands observed and that was at $200 \mathrm{MPa}$, the highest confining pressure tested. Diffuse compaction bands were also observed at this pressure and $150 \mathrm{MPa}$, the next lowest tested. At all lower pressures (50,60, 75, and $90 \mathrm{MPa})$ only high-angle shear bands were observed. For the Rothbach sandstone, diffuse compaction bands were observed at the highest confining pressure tested, $130 \mathrm{MPa}$, and for the Darley Dale, cataclastic flow was observed at the highest pressure, $110 \mathrm{MPa}$. High-angle shear bands were observed at all lower pressures.

[24] Obviously, there is little agreement between the predictions of the transition confining stress and the observations although the absence of observations of compaction bands below the transition pressure in the Darley Dale and Rothbach might constitute some minimal consistency with predictions. There are, however, several possible reasons for the discrepancy. One, already noted, is that the transition pressure was calculated based on parameters of an initial yield surface rather than that at the current inelastic strain level. Another is that the transition pressure is the maximum at which shear bands are predicted but for pressures below this the critical values of $k$ for shear bands and compaction bands are not very different. Consequently, reported observations of compaction bands at confining pressures well below the predicted transition value (for example, for Berea sandstone) could be a combination of compaction and lowangle shear band. In addition, the comparison takes no account of the evolution of $k$ during the test; compaction bands or shear bands might be possible but the value of $k$ may not fall to the critical value needed. Indeed, if normality is satisfied, as is usually assumed for the cap surface, the critical value of $k$ must be nonpositive.

[25] The most likely and most important reason for the discrepancy is that the gross structure of the elliptical surface does not adequately capture the microstructural elements that influence localization. For example, even the porosity, which is clearly important, does not appear in this description, although it might be incorporated as one of the $\alpha_{\kappa}$ and is indirectly incorporated via $P^{*}$. There are, however, indications that the occurrence of compaction bands depends on the nature and uniformity of the microstructure. DiGiovanni et al. [2000] have documented differences in the microstructures of Castlegate and Berea sandstones that may affect the different occurrence and form of compaction bands in these rocks. Numerical simulations by Katsman et al. [2003] suggest that the form and development of compaction bands depend on the degree of disorder in material properties. Baud et al. [2004] discuss other microstructural features that may also affect compaction band formation.

[26] The analysis also shows that the band angle (expressed here as the angle between the normal to the band and the direction of maximum compression) increases continuously from zero (for a compaction band) with decreasing confining stress once shear bands become possible. Although the increase is continuous, it is rapid (see Figures 5-8) until about $20^{\circ}-30^{\circ}$. This is a possible explanation for the uncommon observation of very low angle shear bands, less than $20^{\circ}$. Although the band angle typically decreases with increasing confining stress [Bésuelle and Rudnicki, 2004], values lower than $20^{\circ}$ are seldom reported. For example, for Vosges sandstone, Bésuelle et al. [2000] report a nearly linear decrease in band angle from $55^{\circ}$ to $35^{\circ}$ as the confining pressure increases from 10 to $60 \mathrm{MPa}$. (They report this as an increase in the complement of the angle used here). Note that for band angles of less than $20^{\circ}$, the critical values of $k$ for shear bands and compaction bands are not very different. Thus the slightly undulating appearance of the bands when viewed at the small scale [Wong et al., 2001; Baud et al., 2004] may reflect extension by alternating pure compaction and very low angle shear bands due to local stress alterations. Also, in his early experimental work on compaction bands in Castlegate sandstone, Olsson [1999] observed both compaction bands and shear bands in the same samples summarized in the sketches of his Figure 7. The shear bands appear to be at angles in the range $30^{\circ}-45^{\circ}$ but the sketch for $100 \mathrm{MPa}$ confining pressure and $2.5 \%$ axial strain shows a structure identified as a compaction band with a normal that is slightly inclined (less than $20^{\circ}$ ) to the maximum compression direction.

[27] Compaction bands are observed to form on portions of the stress versus strain curves that are approximately flat (corresponding to $k \approx 0$ ) but may contain one or more episodes of small stress drops followed by some restrengthening. (Once a band or other localized feature forms, the curve does not represent the stress versus strain behavior of a material, but rather the composite behavior of a nonuniform sample.) Olsson and Holcomb [2000], Holcomb and Olsson [2003], and Olsson [2001] observe a small stress drop at the onset of band formation and then a flat or slightly rising stress versus strain curve as the band thickens to encompass the entire length of the sample. Baud et al. [2004] observe several sequential small stress drops followed by restrengthening, each of which appears to correspond to the formation of another discrete band. These observations are roughly consistent with the analysis here in the sense that the maximum value of $k_{\text {crit }}$ is zero (corresponding to a flat stress versus strain curve) or slightly positive, for nonnormality, and that very negative values of $k$ are not required for compaction band formation. On the other hand, compaction bands have not been observed to form on rising portions of the stress versus strain curves (which, at least indirectly, supports the common assumption that normality is satisfied on cap surfaces).

[28] The analysis has focused on a single elliptical cap surface but the behavior of rocks at lower confining stresses is typically described by another "shear" surface (Figure 1). The two surfaces are often interpreted to reflect different microscale mechanisms, for example, microcracking leading to dilation versus pore collapse and indentation leading to compaction, or different deformation styles, for example, brittle behavior and cataclastic flow. Wong et al. [1997, 1992] show that data for shear localization of sandstones at lower mean stresses can be described by a parabolic 
envelope that becomes roughly linear at higher mean stresses. The vicinity of the intersection of the two surfaces is difficult to explore experimentally. The surfaces are usually assumed to intersect with a finite vertex angle, but they are sometimes connected smoothly [e.g., Fossum and Fredrich, 2000a].

[29] Although a rationale for the use of two surfaces is to describe differing regimes of behavior, the nature and evolution of the surfaces is not connected to the microscale mechanisms of deformation in any precise way and they are essentially a phenomenological description. The analysis indicates that the use of two surfaces is not, however, necessary to describe a change in macroscopic failure behavior. The analysis of the elliptic yield surface indicates that there is a transition from shear localization to compaction localization as the lateral confining stress is increased. Although compaction localization is possible for still further increases in confining stress, it is increasingly difficult to achieve because of the increasingly large negative values of $k_{\text {crit }}$ consistent with a transition to diffuse cataclastic flow. Carroll [1991] has also used a one-surface model to describe a transition from dilatant to compactant behavior with increasing mean stress in Boise sandstone. In Carroll's [1991] model, the yield surface is a parabola of fixed width where it intersects the mean stress axis. The height of the parabola increases or decreases as the apex moves along the "critical state line" in the plane of equivalent shear stress and mean stress. The critical state line is so called because, assuming normality, the increment inelastic volume strain is zero for stress states on this line.

[30] Despite the adequacy of a single surface model in some cases, there remain good reasons for a two surface model reflecting brittle deformation and cataclastic flow. Baud et al. [2004] have noted that they observe compaction bands at "stress states that are associated with the transition region from brittle faulting to cataclastic flow" and Wong et al. [2001, p. 2521] noted "our data rule out a constitutive model that does not account for the activation of multiple damage mechanisms in the transitional regimes." Although these statements do not rule out a single surface model with a dependence on different mechanisms, a simple interpretation would be a two-surface model. Conditions for localization at a corner where two surfaces meet are more complicated [Bésuelle and Rudnicki, 2004] but have been analyzed for some special cases by Issen [2002]. In essence, the greater flexibility for the "direction" of the inelastic strain increment vector at a vertex allows the localization condition to be met more easily, albeit at the cost of knowing the parameters for both surfaces.

[31] The analysis here addresses only the initiation of the bands. In the experiments of Baud et al. [2004] and Wong et al. [2001] increasing axial strain causes additional discrete bands to form closer to the center of the specimens. These bands alternate with zones of less compacted material. In contrast, the incipient bands in the experiments of Holcomb and Olsson [2003], Olsson [2001], and Olsson and Holcomb [2000] widen, spread away from the ends of the samples and eventually sweep over the entire sample. Reasons for the differences are not known. In all the experiments, the bands appear (more or less) suddenly across the entire radius of the sample consistent with explanation of their onset as a bifurcation from homogeneous deformation. In the experiments, the length of the bands is, of course, limited to the width of the sample, but in the field, the bands have been observed to be $10 \mathrm{~m}$ or more in length [Mollema and Antonellini, 1996]. Consequently, an understanding of the conditions for extensions of the bands is needed for interpretation of the field observations. To gain insight into the mechanisms for band extension, Vajdova and Wong [2003] have conducted axisymmetric compression tests on circumferentially notched samples. They find that bands initiate at the notch and propagate incrementally as indicated by bursts of acoustic emission activity. More detailed analysis of the band extension will require a better description of the inelastic behavior including the shape of the yield surface and its evolution with inelastic deformation.

\section{Conclusion}

[32] The analysis of conditions for shear and compaction localization on an elliptical cap yield surface reveals that there is a point on the ellipse, corresponding to a specific lateral confining pressure in the axisymmetric compression test, where there is a transition from shear band formation to compaction band formation. This point also corresponds to the maximum value of $k_{\text {crit }}$, the slope of the mean compression stress versus inelastic volume strain curve required for localization. This maximum value is zero if normality is satisfied but slightly positive if it is not. For confining stresses higher than that corresponding to the transition, compaction bands are the only localized mode possible but $k_{\text {crit }}$ decreases (becomes more negative) with increasing confining stress. For lower values, both shear bands and compaction bands are possible but $k_{\text {crit }}$ for shear bands is greater than that for compaction bands suggesting that shear bands will occur first. These results are consistent with observations of compaction band formation in experiments on relatively flat portions of the stress versus strain curve, corresponding to $k_{\text {crit }} \approx 0$, in a limited range of lateral confining stresses, and in a transition region between brittle faulting behavior and cataclastic flow. The predicted angle between the normal to the shear band and specimen axis increases continuously but rapidly from zero, corresponding to compaction bands, to about $20^{\circ}-30^{\circ}$. This result is consistent with the infrequent observation of shear bands inclined at large angles to the specimen axis in triaxial compression tests.

[33] Acknowledgments. I am grateful to Teng-fong Wong for many stimulating discussions and for his generosity in providing manuscripts and data prior to publication. In addition, in his review he pointed out an algebraic error in my interpretation of data. Other review comments by Teng-fong and Joanne Frederich substantially improved the manuscript. I have also benefited from discussions with Eric Grueschow, David Holcomb, Kathleen Issen, and Bill Olsson. Partial financial support for this work was provided by the U.S. Department of Energy, Office of Basic Energy Science, Geosciences Research Program through grant DE-FG-0293ER14344 to Northwestern University.

\section{References}

Antonellini, M., and A. Aydin (1994), Effect of faulting on fluid flow in porous sandstones: Petrophysical properties, Am. Assoc. Pet. Geol. Bull., $78,355-377$

Antonellini, M., and A. Aydin (1995), Effect of faulting on fluid flow in porous sandstone: Geometry and spatial distribution, Am. Assoc. Pet. Geol. Bull., 79, 642-671. 
Antonellini, M. A., A. Aydin, and P. P. Pollard (1994), Microstructure of deformation bands in porous sandstones at Arches National Park, Utah, J. Struct. Geol., 16, 941-959.

Aydin, A., and A. M. Johnson (1983), Analysis of faulting in porous sandstones, J. Struct. Geol., 5, 10-31.

Bastawros, A.-F., H. Bart-Smith, and A. G. Evans (2000), Experimenta analysis of deformation mechanisms in a closed-cell aluminum alloy foam, J. Mech. Phys. Solids, 48, 301-322.

Baud, P., E. Klein, and T.-F. Wong (2004), Compaction localization in porous sandstones: Spatial evolution of damage and acoustic emission activity, J. Struct. Geol., in press.

Bésuelle, P., and J. W. Rudnicki (2004), Localization: Shear bands and compaction bands, in Mechanics of Fluid Saturated Rocks, edited by Y. Guéguen and M. Boutéca, Academic, San Diego, Calif., in press.

Bésuelle, P., J. Desrues, and S. Raynaud (2000), Experimental characterisation of the localisation phenomenon inside a Vosges sandstone in a triaxial cell, Int. J. Rock Mech. Min. Sci., 37, 1123-1237.

Carroll, M. M. (1991), A critical state plasticity theory for porous reservoir rock, in Recent Advances in Mechanics of Structured Continua, vol. 117 edited by M. Massoudi and K. R. Rajagopal, pp. 1-8, Am. Soc. of Mech. Eng., New York.

Chapin, J. R., K. R. Sternlof, D. D. Pollard, and L. J. Durlofsky (2004), Permeability effects of systematic deformation band arrays in sandstone, Am. Assoc. Pet. Geol. Bull., in press.

DiGiovanni, A. A., J. T. Fredrich, D. J. Holcomb, and W. A. Olsson (2000), Micromechanics of compaction in an analogue reservoir sandstone, in Pacific Rocks 2000, Proceedings of the 4th North American Rock Mechanics Symposium, edited by J. Girard et al., pp. 1153-1158, A. A. Balkema, Brookfield, Vt.

Dimaggio, F. L., and I. S. Sandler (1971), Material model for granular soils, J. Eng. Mech. Div. Am. Soc. Civ. Eng., 97, 935-950.

Fossum, A. F., and J. T. Fredrich (1998), Estimation of constitutive parameters for the Belridge diatomite, South Belridge diatomite field, Tech. Rep. SAND98-1407, Sandia Nat. Lab., Albuquerque, N. M.

Fossum, A. F., and J. T. Fredrich (2000a), Cap plasticity models and compactive and dilatant pre-failure deformation, in Pacific Rocks 2000 Proceedings of the 4th North American Rock Mechanics Symposium edited by J. Girard et al., pp. 1169-1176, A. A. Balkema, Brookfield, $\mathrm{Vt}$

Fossum, A. F., and J. T. Fredrich (2000b), Constitutive models for the Etchegoin sands, Belridge diatomite, and Overburden formations at the Lost Hills oil field, California, Tech. Rep. SAND2000-0827, Sandia Nat Lab., Albuquerque, N. M.

Fossum, A. F., P. E. Senseny, T. W. Pfeifle, and K. D. Mellegard (1995), Experimental determination of probability distributions for parameters of a Salem limestone cap plasticity model, Mech. Mater., 21, 119137

Grueschow, E. G., and J. W. Rudnicki (2003), Modeling constitutive behavior and compaction localization for high porosity sandstone, Eos Trans. $A G U, 84(46)$, Fall Meet. Suppl., Abstract T42A-0279.

Gu, C., M. Kim, and L. Anand (2001), Constitutive equations for metal powders: Application to powder forming processes, Int. J. Plast., 17, $147-209$.

Haimson, B. C. (2001), Fracture-like borehole breakouts in high-porosity sanstone: Are they caused by compaction bands?, Phys. Chem. Earth, Part A, 26, 15-20.

Holcomb, D. J., and W. A. Olsson (2003), Compaction localization and fluid flow, J. Geophys. Res., 108(B6), 2290, doi:10.1029/2001JB000813.

Issen, K. A. (2002), The influence of constitutive models on localization conditions for porous rock, Eng. Fract. Mech., 69, 1891-1906.

Issen, K. A., and J. W. Rudnicki (2000), Conditions for compaction bands in porous rock, J. Geophys. Res., 105, 21,529-21,536.

Issen, K. A., and J. W. Rudnicki (2001), Theory of compaction bands in porous rock, Phys. Chem. Earth, Part A, 26, 95-100.
Katsman, R., E. Aharonov, and H. Scher (2003), Analysis of interaction between compaction and cracking in high-porosity rock (abstract), Geophys. Res. Abstr. [CD-ROM], 5

Kim, M. K., and P. V. Lade (1984), Modeling rock strength in three dimensions, Int. J. Rock Mech. Min. Sci., 21, 21-33.

Kirby, S., W. B. Durham, and L. Stern (1992), The ice I-II transformation: Mechanisms and kinetics under hydrostatic and nonhydrostatic conditions, in Physics and Chemistry of Ice, edited by N. Maeno and T. Hondoh, pp. 456-462, Hokkaido Univ. Press, Sapporo, Japan.

Klaetsch, A. R., and B. C. Haimson (2002), Porosity-dependent fracturelike breakouts in St. Peter sandstone, in Mining and Tunneling Innovation and Opportunity, edited by R. Hammah et al., pp. 1365-1371, Univ. of Toronto Press, Canada.

Klein, E., P. Baud, T. Reuschlé, and T.-F. Wong (2001), Mechanical behaviour and failure mode of Bentheim sandstone under triaxial compression, Phys. Chem. Earth, Part A, 26, 21-25.

Lee, D.-H., C. H. Juang, J.-W. Chen, H.-M. Lin, and W.-H. Shieh (1999), Stress paths and mechanical behavior of a sandstone in hollow cylinder tests, Int. J. Rock Mech. Min. Sci., 36, 857-870.

Mollema, P., and M. A. Antonellini (1996), Compaction bands: A structural analog for anti-mode I cracks in aeolian sandstone, Tectonophysics, 267, $209-228$

Olsson, W. A. (1999), Theoretical and experimental investigation of compaction bands in porous rock, J. Geophys. Res., 104, 7219-7228.

Olsson, W. A. (2001), Quasistatic propagation of compaction fronts in porous rocks, Mech. Mater., 33, 659-668.

Olsson, W. A., and D. J. Holcomb (2000), Compaction localization in porous rock, Geophys. Res. Lett., 27, 3537-3540.

Ottosen, N. S., and K. Runesson (1991), Properties of discontinuous bifurcation solutions in elasto-plasticity, Int. J. Solids Struct., 27, 401-421.

Papka, S. D., and S. Kyriakides (1998), Experiments and full-scale numerical simulations of in-plane crushing of a honeycomb, Acta Mater., 46, $2765-2776$.

Park, C., and S. R. Nutt (2001), Anisotropy and strain localization in steel foam, Mater. Sci. Eng. A, 299, 68-74.

Perrin, G., and J. B. Leblond (1993), Rudnicki and Rice's analysis of strain localization revisited, J. Appl. Mech., 60, 842-846.

Rudnicki, J. W. (2002), Conditions for compaction and shear bands in a transversely isotropic material, Int. J. Solids Struct., 39, 3741-3756.

Rudnicki, J. W. (2003), Compaction bands in porous rock, in Bifurcations and Instabilities in Geomechanics, edited by J. F. Labuz and A. Drescher, pp. 29-39, A. A. Balkema, Brookfield, Vt.

Rudnicki, J. W., and J. R. Rice (1975), Conditions for the localization of deformation in pressure-sensitive dilatant materials, J. Mech. Phys. Solids, 23, 371-394.

Schofield, A. N., and P. Wroth (1968), Critical State Soil Mechanics, McGraw-Hill, New York.

Vajdova, V., and T.-F. Wong (2003), Incremental propagation of discrete compaction bands: Acoustic emission and microstructural observations on circumferentially notched samples of Bentheim, Geophys. Res. Lett., 30(14), 1775, doi:10.1029/2003GL017750.

Wong, T.-F., H. Szeto, and J. Zhang (1992), Effect of loading path and porosity on the failure mode of porous rocks, Appl. Mech. Rev., 45, $281-$ 293.

Wong, T.-F., C. David, and W. Zhu (1997), The transition from brittle faulting to cataclastic flow in porous sandstones: Mechanical deformation, J. Geophys. Res., 102, 3009-3025.

Wong, T.-F., P. Baud, and E. Klein (2001), Localized failure modes in a compactant porous rock, Geophys. Res. Lett., 28, 2521-2524.

J. W. Rudnicki, Department of Civil and Environmental Engineering, Northwestern University, Evanston, IL 60208-3109, USA. (jwrudn@ northwestern.edu) 